\title{
Competencias Digitales:
}

\section{Realidad de ingreso de los estudiantes a la vida universitaria}

\author{
Dra. Paola Ascencio Ojeda \\ paola.ascencio@umag.cl \\ Universidad de Magallanes, Chile \\ Dr. Leonardo Glasserman Morales \\ glasserman@tec.mx \\ Tecnológico de Monterrey, México \\ Dr. Jordi Quintana Albalat \\ jordi.quintana@ub.edu \\ Universidad de Barcelona, España
}

\section{Resumen}

El presente estudio pretende revelar las condiciones de entrada con las cuales ingresan los estudiantes a la vida universitaria respecto a la adquisición de las competencias digitales, declaradas como una competencia clave para insertarse en sociedad por varias instituciones gubernamentales y académicas, dada la globalización y dinamización de la sociedad facilitada por la red global de internet, la cual ha modificado todas las formas de comunicación, socialización y operación de los servicios más básicos de la cotidianeidad. Por ello no debe faltar en el desarrollo de saberes, habilidades y actitudes de todo ser humano, siendo entendida como una herramienta imprescindible para toda la ciudadanía. La Universidad de Magallanes en Chile, ha instaurado desde el año 2010 para todos los estudiantes que ingresan a primer año, en cualquiera de sus carreras profesionales, la aplicación de una Prueba de Suficiencia en Computación, la cual tiene como objetivo validar las habilidades y saberes relativos a las Tecnologías de Información y la Comunicación de los estudiantes, basándose básicamente en la propuesta que el sistema educativo Chileno promueve en este orden para la enseñanza básica y media, diseñada por el Centro de Educación y Tecnologías - Red Enlaces del Ministerio de Educación de Chile, para así reconocer cuáles son las competencias adquiridas durante ese proceso, y otorgando la posibilidad de valorar saberes previos, y eximirse de cursar una asignatura, en caso de aprobación. El objetivo principal de la prueba, en el contexto de ésta investigación, es saber si realmente los estudiantes ingresan a la vida universitaria con competencias digitales instaladas, si se encuentran formados o preparados en este ámbito, y por otra parte tiene que ver con conocer los ámbitos con áreas de oportunidad que se detecten a partir de la evaluación y que tengan relación primeramente con la Matriz de Habilidades TIC para el aprendizaje y las consideradas de experiencias de otras universidades e instituciones del mundo, que permita gestar comentarios y sugerencias para potenciarlas en el proceso formativo universitario.

\section{Palabras Clave}

Competencias clave; competencia digital; universidad; TIC; formación. 


\title{
Digital Competences:
}

\section{Reality of students starting university life}

\author{
Dra. Paola Ascencio Ojeda \\ paola.ascencio@umag.cl \\ Universidad de Magallanes, Chile
}

\section{Dr. Leonardo Glasserman Morales}

\author{
glasserman@tec.mx \\ Tecnológico de Monterrey, México

\section{Dr. Jordi Quintana Albalat} \\ jordi.quintana@ub.edu \\ Universidad de Barcelona, España
}

\begin{abstract}
This study aims to reveal the conditions of entry with which students start university life with respect to the acquisition of digital skills, declared as a key competence to be inserted into society by various governmental and academic institutions, given the globalization and dynamization of the society facilitated by the global internet network, which has modified all forms of communication, socialization and operation of the most basic services of everyday life. Therefore, it must not be lacking in the development of knowledge, skills and attitudes of every human being, being understood as an essential tool for all citizens. The University of Magallanes in Chile, has established since 2010 for all students entering the first year of undergraduate studies the application of a Computer Proficiency Test, which aims to validate skills and knowledge related to the Information and Communication Technologies of the students. Based basically on the proposal that the Chilean education system promotes in this order for basic and secondary education, designed by the Center for Education and Technologies - "Red Enlaces" of the Chilean Ministry of Education, in order to recognize what are the skills acquired during this process, and granting the possibility of assessing previous knowledge, and exempting oneself from taking a subject, in case of approval. The main objective of the test, in the context of this research, is to know if students really start university life with digital skills installed, if they are trained or prepared in this area, and on the other hand it has to do with knowing the areas of opportunity that are detected from the evaluation and that are primarily related to the Matrix of ICT Skills for learning and those considered from experiences of other universities and institutions in the world, which allow to generate comments and suggestions to enhance them in the university training process.
\end{abstract}

\section{Keywords}

Key competences; digital competence; university; ICT; training 


\section{Introducción}

Hoy en día la sociedad digital, cuenta con un andamiaje basado en la capacidad que la ciudadanía debe de alcanzar para identificar, recuperar, procesar, transformar, reelaborar, producir, utilizar, difundir y compartir información, orientada a generar y aplicar conocimientos necesarios para el aprendizaje, el desarrollo del conocimiento y la cultura, y la cohesión social, entendiendo que la sociedad fundamenta su éxito actual en estas acciones. Esto obliga a los actores de las diversas estructuras sociales, económicas, culturales, educativas, entre otras, a gestar sugerencias, políticas y prácticas necesarias para adaptarse y desenvolverse en estas nuevas formas de organización. Y de esta forma, obtener mejores oportunidades de inserción, éxito e innovación, donde las Tecnologías de Información y la Comunicación (TIC) median y lideran los procesos del cambio.

Las tecnologías digitales están presentes en toda actividad humana de la actualidad ya que se han convertido en una necesidad, instalándose como un saber y unas habilidades implícitas para el desarrollo de la ciudadanía; surgiendo hoy como un requerimiento y competencia clave. En este sentido, la adquisición de información como base para la construcción de un conocimiento por medio de las competencias informacionales, se presenta como una de las claves para el éxito de las sociedades emprendedoras. En Chile y el mundo, un ciudadano no debe estar exento de ellas. Es por esta razón, que el sistema educativo, pilar fundamental de los cambios esperados en una sociedad, ha integrado en sus propios procesos, tanto en la gestión administrativa como docente, los recursos y habilidades TIC, siendo difícil en la actualidad evitar pensar la educación y sus procesos, sin la inclusión de éstas.

Asumiendo esta realidad, a partir del año 2008, el Ministerio de Educación de Chile comienza a diseñar y construir una serie de referencias en esta línea, materializadas en un mapa de progreso de habilidades TIC funcionales para estudiantes, denominado Estándar K-12 y una Matriz de Habilidades TIC para estudiantes del siglo XXI (Ministerio de Educación, 2012). Ambos han sido utilizados para el desarrollo del Sistema Nacional de Medición de Competencias TIC en Estudiantes (SIMCE TIC) por parte del Ministerio de Educación (2013b), aplicado por primera vez a nivel nacional en el mes de noviembre del año 2011 (ENLACES, 2015). La Matriz generada el año 2008, fue sometida durante 2012 a un proceso de actualización y validación. Dicho reajuste se relaciona fundamentalmente con los cambios sociales impulsados en la educación y sigue la dinámica de las actualizaciones que otros países e instituciones han desarrollado. El proceso consistió en la revisión de antecedentes relevantes para el diseño de una matriz que considere los principales cambios ocurridos en los últimos años, tanto a nivel internacional como nacional, en relación con la definición, desarrollo y medición de habilidades TIC en el contexto escolar.

En consecuencia, surge finalmente la Matriz de Habilidades TIC para el Aprendizaje (HTPA), diseñada por el Ministerio de Educación (2013a) en el marco de la asesoría de actualización y validación de la Matriz de Habilidades TIC para estudiantes. Esta pretende ser considerada en los procesos formativos y desarrollar en los alumnos de los niveles educativos de enseñanza básica y media, la capacidad para desenvolverse adecuadamente en los ámbitos de trabajo con información y comunicación en ambiente digital, definida como "la capacidad del estudiante de resolver problemas de información, comunicación y conocimiento, así como dilemas éticos en ambiente digital" (Ministerio de Educación, 2013a, p. 21).

Por otra parte, al considerar el concepto genérico sobre competencia digital, este tiene relación con el dominio de diferentes procesos y estrategias, o sistemas de aptitudes, que pueden permitir que una persona se desenvuelva cómodamente en una actividad de la sociedad actual, mediante el uso transversal de las TIC. En otras palabras, son los conocimientos que están asociados a la comprensión 
de las innumerables oportunidades que las nuevas tecnologías ofrecen, destacando el uso de recursos apropiados para producir, presentar o comprender información compleja, la búsqueda y selección de información necesaria para una actividad, la utilización de las TIC para apoyar el pensamiento crítico en cuanto a su uso en forma autónoma, reflexiva, positiva y de la sensibilidad que se debe tener por la responsabilidad que demandan las tecnologías.

De acuerdo a todo lo anterior, en la Universidad de Magallanes (UMAG) de la Patagonia Chilena se considera dentro de sus grandes tareas, y así lo indica el Proyecto Educativo Institucional de Universidad de Magallanes (2010c), el diseño, construcción y aplicación, de una prueba de suficiencia, que procede para eximirse (en caso de aprobación) de la asignatura institucional "Computación Básica" de la Universidad de Magallanes (2014), asociada a esta competencia, la cuál es aplicada a todos los estudiantes que ingresan por primera vez a la vida universitaria, donde ellos colocan a pruebas sus conocimientos y competencias, mediante la resolución de un caso que transita por los ámbitos definidos de competencia digital, entendiendo que la única forma de conocer realmente el logro de una tarea, es poniendo a prueba los conocimientos, manejos, actitudes del estudiante frente a un problema real y contextualizado.

El objetivo de la presente investigación se fundamenta mostrar el escenario real desde donde se sitúan los estudiantes al llegar a educación superior respecto de sus competencias digitales, y de esta forma, comentar y sugerir acciones posteriores orientadas a fortalecer aquellas falencias detectadas durante el proceso investigativo, dando valor a los procesos formativos de la UMAG (asignatura institucional "Computación Básica"). Por otra parte, y en coherencia con lo anterior, se pretende dar respuesta a la siguiente pregunta: ¿qué tan preparados se encuentran los estudiantes que ingresan a la vida universitaria en la Universidad de Magallanes en Chile respecto de las competencias digitales?

Finalmente, esta medición considera referentes teóricos para su construcción, levantados del contexto tanto nacional como internacional, dando vida a una prueba que configura ambos sistemas educativos, el de procedencia (educación media) y el de referencia (educación superior), donde la convergencia de saberes esperados en cuanto, a las TIC y sus usos, debe darse para lograr la aprobación, además de ofrecer datos para el análisis de la realidad, fundamento del presente estudio.

\section{Marco Teórico}

Las TIC y el internet son protagonistas indiscutibles de los cambios sociales. Su incorporación en todos los frentes de las actividades cotidianas ha resignificado el quehacer humano y su comportamiento. Es así como el nivel de importancia que han alcanzado las TIC y su transversalidad han significado para los ciudadanos una serie de transiciones y cambios que inciden fundamentalmente en cómo se establecen relaciones el día de hoy en los distintos ámbitos en los cuáles nos desarrollamos. Todo ello gracias a que la globalización entrega nuevas formas de comunicación, vorágine de información y conocimiento disponible en esta nueva sociedad.

Para lograr entender y establecer cuáles serían los criterios, ámbitos o dominios a evaluar en los estudiantes ingresados a la universidad, lo primero ha sido seleccionar definición sobre competencia digital, que actualmente lidera al mundo del trabajo y el universitario, que pertenece a la Comisión Europea y que opera para todo sistema, la cual se define como: 
La competencia digital entraña el uso seguro y crítico de las Tecnologías de la Información Sociedad (TSI) para el trabajo, el ocio y la comunicación. Se sustenta en las competencias básicas en TIC: el uso de ordenadores para obtener, evaluar, almacenar, producir, presentar e intercambiar información, y comunicarse y participar en redes de colaboración a través de Internet (Parlamento Europeo y Consejo de la Unión Europea, 2006).

Otras propuestas son las de Ala-Mutka (2011), quien menciona que la competencia digital puede ser definida como el uso seguro, crítico y creativo de las TIC para lograr objetivos relacionas con el trabajo, la empleabilidad, el aprendizaje, el ocio, la inclusión y la participación en la sociedad, o la de Ferrari (2012), en relación a las competencias digitales para la ciudadanía, que fueron actualizadas en la versión 2.1 (Carretero, Vuorikari y Punie, 2017), que considera que un ciudadano considerado digitalmente competente significa que debe tener capacidad de comprender los medios digitales para buscar información, ser crítico sobre lo que se recupera y poder comunicarse con otros utilizando una variedad de herramientas y aplicaciones digitales.

Con estas definiciones, se han identificado diferentes experiencias internacionales que han permitido definir cuáles son las dimensiones, áreas o ámbitos que no deben faltar en la medición de una competencia digital (contextualizada en el presente estudio) considerando sin lugar a duda así también, lo que sugiere el gobierno chileno en su definición de Habilidades TIC para el aprendizaje: "'La capacidad de resolver problemas de información, comunicación y conocimiento, así como dilemas legales, sociales y éticos en ambiente digital" (Ministerio de Educación de Chile, 2013).

Y finalmente la competencia sello propia de la Universidad de Magallanes "Habilidades en el uso de tecnologías de la información y comunicación", la cual no considera una definición, pero si establece niveles de desempeño:

- Nivel 1 de Desempeño: Crea y administra correctamente archivos, generando documentos con procesador de textos, planillas de cálculo y programas de presentaciones; Navega en Internet y utiliza correctamente el correo electrónico.

- Nivel 2 de Desempeño: Utiliza y combina distintos programas como procesador de texto, planillas de cálculo, plantillas de presentación, y dispositivos periféricos, para desarrollar productos multimediales de mediana complejidad.

- Nivel 3 de Desempeño: Utiliza las habilidades desarrolladas en este ámbito, como base para construir nuevos conocimientos para el uso de las Tecnologías de la Información y Comunicación.

Con todo lo anterior, luego de una revisión documental y bibliográfica extensa (Ala-Mutka, 2011; AlaMutka, Punie y Redecker, 2008; Carretero, Vuorikari y Punie, 2017; Ferrari, 2012; Ilomäki, Paavola, Lakkala y Kantosalo, 2016; Parlamento Europeo y Consejo de la Unión Europea, 2006; SánchezAntolín, Andrés-Vilora y Paredes-Labra, 2018; Villa y Poblete, 2011, entre otros), referenciada por los conceptos previos, es que se ha logrado definir y levantar las cinco dimensiones más adecuadas a ser consideradas para la evaluación o prueba de suficiencia de los alumnos de primer ingreso a la vida universitaria en la UMAG, las cuales son:

D1. Dimensión Cultura y Civismo Digital: Capacidad para entender y desarrollarse de manera efectiva como ciudadano de la sociedad digital. Esto implica, tanto reconocer como utilizar los servicios y recursos disponibles en el mundo digital, para aprender y resolver problemas de la vida personal, académica y luego desempeñarse en el ámbito laboral. 
D2. Dimensión Gestión de la Información: Capacidad para generar y compartir información en diversos formatos dispuestos en la sociedad digital, para aprender y resolver problemas de la vida personal, académica y luego desempeñarse en el ámbito laboral.

D3. Dimensión Comunicación Digital: Capacidad para entender la dinámica actual de la comunicación, así como también comunicarse, relacionarse y colaborar de forma eficiente con herramientas disponibles en los entornos digitales; para aprender en la vida personal, académica y luego desempeñarse en el ámbito laboral.

D4. Dimensión Trabajo en Red: Capacidad para entender los procesos de construcción colaborativa en red, como también trabajar, colaborar y cooperar de manera responsable y eficiente en los entornos digitales, para aprender y resolver problemas de la vida personal, académica y luego desempeñarse en el ámbito laboral.

En coherencia con lo anterior, comentar que todos los documentos revisados explicitan que la inserción y adaptación en sociedad implica la fusión y desarrollo de un conjunto de dominios y todos estos relacionados con las tecnologías, producto de los últimos sucesos y ejercicios que imprime la sociedad y que se entienden como "habilidades para la vida", consideradas como saberes básicos y propios del andamio de la ruta del aprendizaje e inserción social, es entonces como ésta se ha convertido en "una exigencia y un derecho" (OCDE, 2010).

Por otro lado, la misma OCDE (2010) asegura que el desarrollo social y económico exige que los sistemas educativos ofrezcan nuevas habilidades y competencias, que les permitan beneficiarse de las nuevas formas emergentes de socialización y contribuyan activamente al desarrollo económico bajo un sistema cuya principal base es el conocimiento. Así también, es posible indicar que el informe de seguimiento de la iniciativa mundial Educación para Todos (EPT) "Situación Educativa de América Latina y el Caribe: Hacia la educación de calidad para todos al 2015" (UNESCO, 2017) remarca que la formación de competencias digitales es cada vez más importante en el ámbito educativo como una necesidad para la inclusión en la sociedad del conocimiento: las TIC no son solo un potente recurso para el aprendizaje, son herramientas cada vez más relevantes para la vida.

\section{Metodología}

El estudio que se presenta corresponde a una investigación contextualizada en la Universidad de Magallanes en la región de la Patagonia Chilena, donde mediante una prueba de suficiencia se buscó evaluar las competencias digitales adquiridas por estudiantes universitarios. Entre algunas de las que se incluyen se encentran el manejo de las herramientas tecnológicas a disposición, sumado a la aptitud para enfrentar y resolver problemas de diversa complejidad cognitiva, relacionarse con otros y desenvolverse de forma ética y ajustada al marco legal dentro de ambientes tecnológicos. Esto se traduce en la capacidad para resolver problemas y tareas reales en un ambiente virtual, a través del uso de aplicaciones computacionales regularmente utilizadas.

Para seguir avanzando, la presente investigación asume un enfoque fundamentalmente naturalista e interpretativo, paradigma que es también denominado fenomenológico, de acuerdo con Guba (1981). Este tipo de investigación elegida es más abierta y menos restringida, además de permitir abordar más en comparación a otras expresiones dadas, entendiendo que estudia a los objetos y seres vivos en sus contextos o ambientes naturales y cotidianidad, e interpretativo porque intenta encontrar sentido a los 
fenómenos en función de los significados que las personas les otorguen (Hernández, Fernández y Baptista, 2014), es un primer acercamiento a la problemática, para luego poder investigar con mayor profundidad el fenómeno de así requerirlo, utilizando otras modalidades de investigación más pertinentes al objetivo de estudio.

El diseño metodológico posibilita la realización de un plan de trabajo, con estrategias que buscan responder al objetivo de investigación, y de este modo obtener información concreta, fidedigna e interpretable.

En cuanto a la muestra, se ha optado por una de tipo no probabilística e intencional, también llamado de conveniencia o basada en criterios que, según Sabariego (2004, p. 148), es aquella en la que "se seleccionan sujetos particulares que son expertos en un tema o relevantes como fuentes importantes de información según criterios establecidos previamente", porque se intenciona la muestra a casos que aseguren aportar datos que completen y se enfoquen en la búsqueda e interpretación de la realidad.

\section{Caracterización de los estudiantes evaluados}

Los estudiantes evaluados en la presente prueba de suficiencia son provenientes de todas las facultades de la Universidad de Magallanes, estudiantes de carreras profesionales de la oferta formativa entregada por la institución y que ingresan por primera vez a la vida universitaria.

La muestra definitiva de estudiantes con resultados válidos para la prueba de suficiencia alcanzó un total de 405 participantes, de los cuáles es posible observar e indicar a partir de la siguiente infografía y los resultados obtenidos que, un $57,04 \%$ de los alumnos que han participado de la aplicación, proviene de un sistema de dependencia administrativa Subvencionado (con un porcentaje de aporte del estado), presentando una mayoría absoluta respecto de la variable Procedencia. En la infografía 1 se presenta gráficamente la distribución de estos participantes.

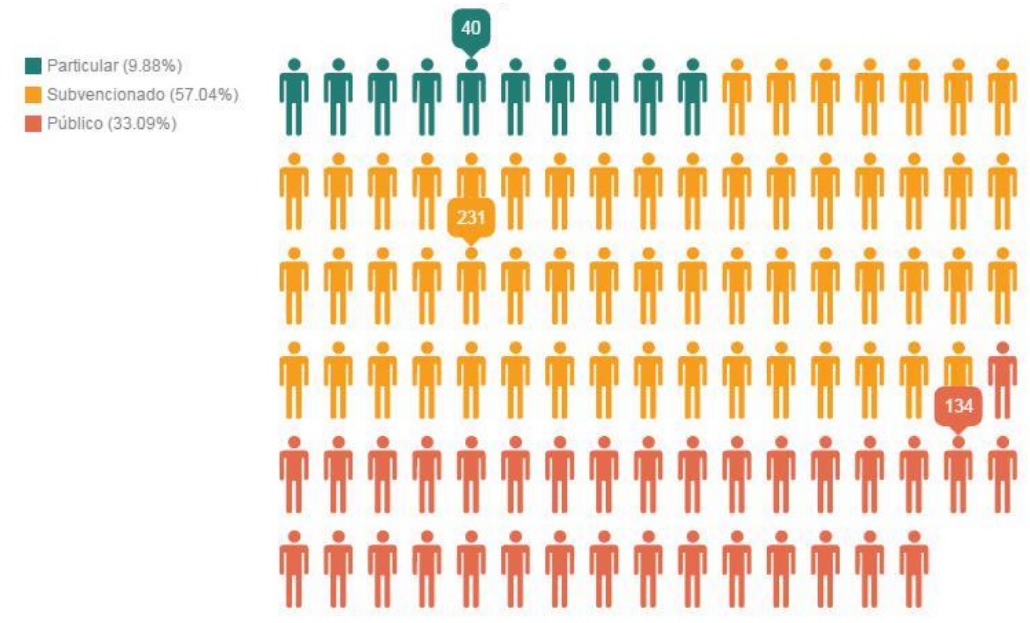

Infografía. 1. Distribución por procedencia 
De acuerdo con el gráfico n. ${ }^{\circ} 1$ que se presenta a continuación, la edad media de los alumnos que fueron encuestados es de 19 años y las edades en las que oscilan los participantes están entre 17 a 39 años.

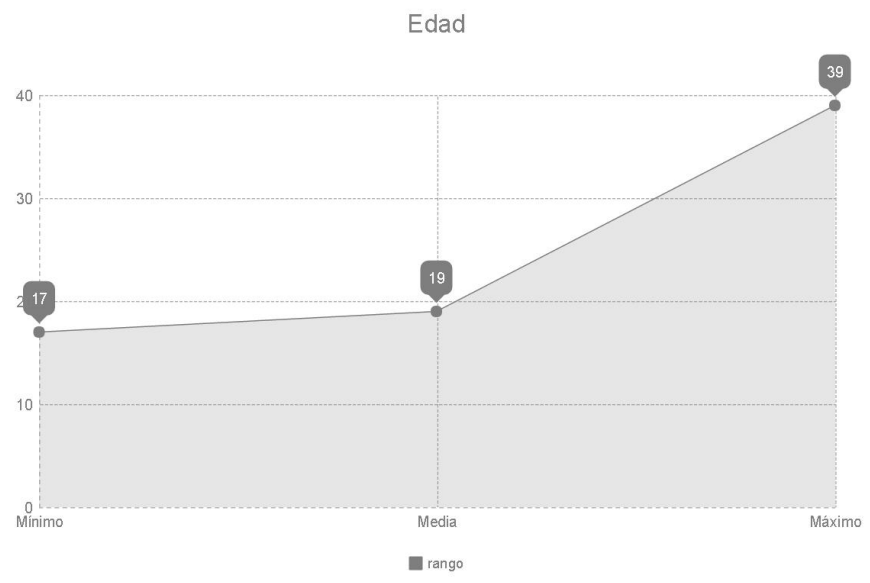

Gráfico. 1. Edad de los evaluados

De los evaluados, el $66.91 \%$ corresponde a estudiantes del sexo femenino, con una frecuencia de 271 , mientras que el $33.09 \%$ de la muestra corresponde a personas del sexo masculino con una frecuencia ponderada en 134.

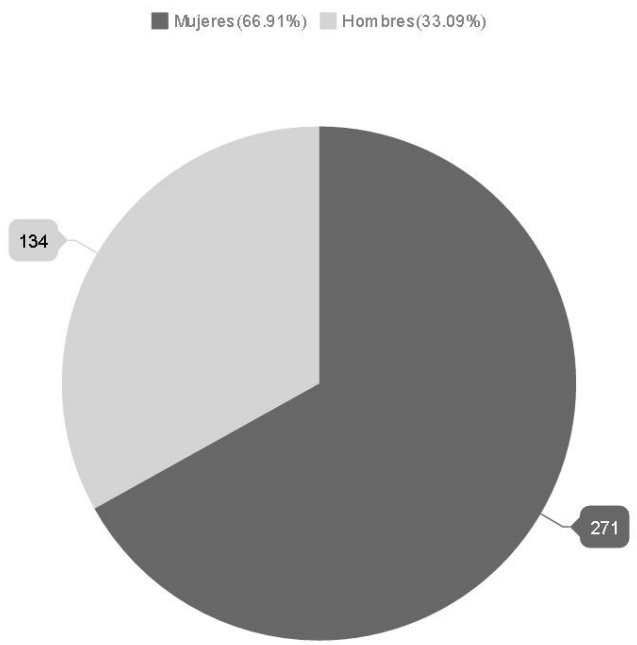

Gráfico. 2. Distribución por sexo 


\section{Resultados de la prueba de suficiencia}

Se presentan resultados institucionales que dan cuenta de las ponderaciones y respuestas asociadas a la prueba de suficiencia en computación aplicada a los estudiantes de la casa de estudios. De esta manera se puede observar y conocer datos acerca de los ámbitos más desfavorecidos y carentes en nuestros estudiantes relacionados a las TIC.

Parece adecuado comentar que la corrección de la prueba considera la utilización de distintas estrategias evaluativas, en función de los ítems que la componen. De modo que las situaciones planteadas son corregidas por el docente que tiene en cuenta las herramientas que ha utilizado el estudiante para movilizar sus conocimientos y ponerlos en práctica. Los productos son revisados en función de una rúbrica de corrección, por individuos especialmente capacitados para este propósito, los cuáles han sido supervisados por los docentes de la asignatura.

Los ámbitos son evaluados independientemente con un nivel de exigencia distinta para cada una, debiendo alcanzar una ponderación de 4.0 para aprobar (en escala evaluativa del sistema escolar chileno). Esta nota final considera el resultado de cada ámbito ya mencionado con sus respectivos pesos evaluativos.

Las figuras que se presenta a continuación muestran cinco ámbitos, de los cuales, cuatro han sido homologados con los de la Matriz HTPA y uno que refleja lo demás, las actualizaciones levantadas desde las experiencias internacionales. De esta manera, se gestan las analogías necesarias que permitan obtener los datos esperados.

\section{a. Resultados}

Se parte dando cuenta del número de estudiantes que aprueba o reprueba la asignatura en función de la obtención de la nota mínima 4.0. Esto en conjugación con los pesos y ponderaciones explicadas anteriormente, para luego de ello pasar por un análisis descriptivo asociado a las respuestas y ámbitos. 


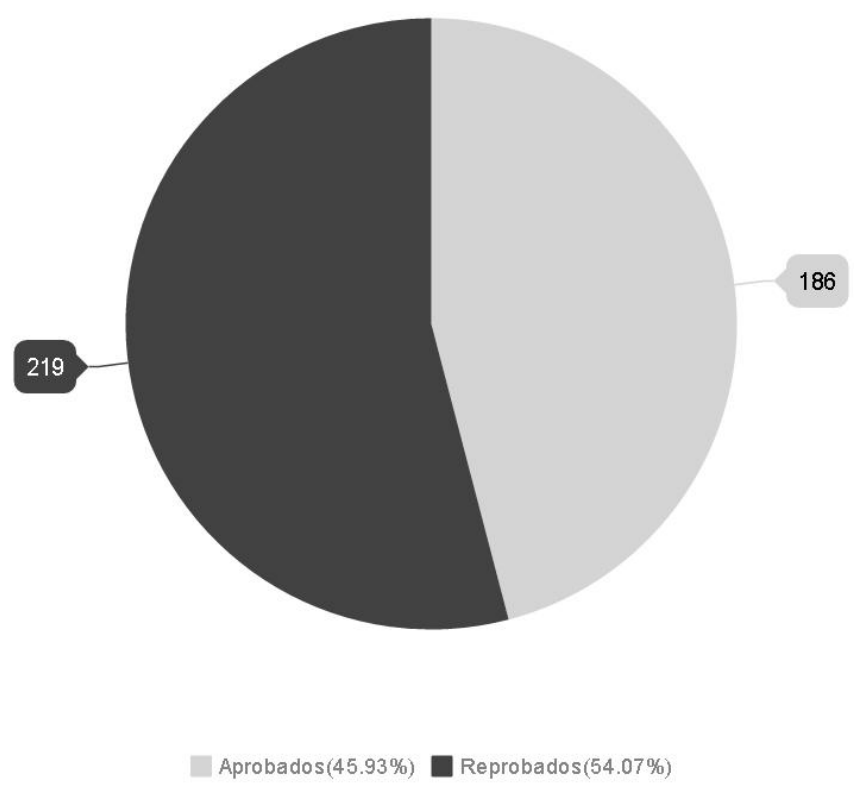

Gráfico. 3. Aprobación/Reprobación

El gráfico n.० 3 muestra que el $45.93 \%$ de los estudiantes evaluados supera la prueba y, por tanto, tienen la oportunidad de no cursar la asignatura y mantener la valoración obtenida o lograda durante la prueba y revela que el número de estudiantes aprobados es inferior al de reprobados, existía la idea de que sería diferente, entendiendo que muchos de los dominios evaluados son considerados en la Matriz HTPA, por lo cual deberían de haberse anclado durante el proceso formativo antecesor y en educación formal, según se establece como política y lineamiento en Chile, además de las recomendaciones de estudio y práctica entregadas a los estudiantes por parte de la institución, para desarrollar de manera cómoda la prueba institucional.

A partir del establecimiento educacional de procedencia de los estudiantes recibidos por la institución y en relación con el tipo de administración de los mismos, es posible inferir a partir de los datos, que no hay diferencias significativas en cuanto a las habilidades TIC instaladas. Al menos las notas alcanzadas no ofrecen señales para este segmento de que la educación particular pagada haga entrega de conocimientos y actitudes más acabadas en cuanto a las habilidades en TIC que debieran lograrse de acuerdo con las políticas chilenas. Como es posible observar, las variaciones de puntajes son mínimas. 


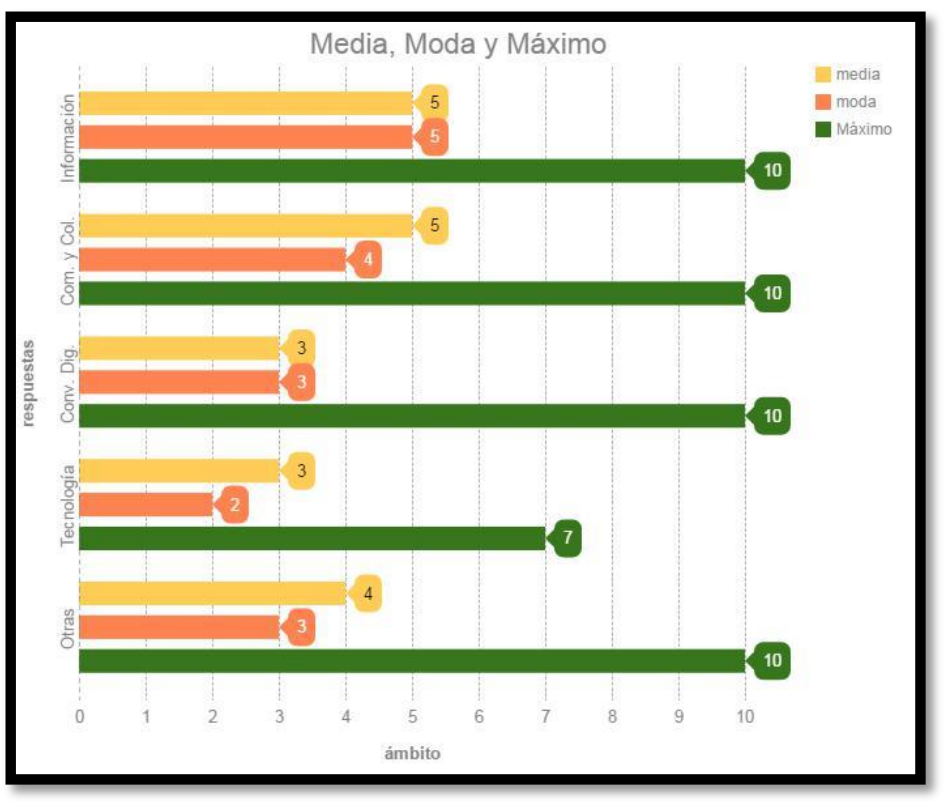

Gráfico. 4. Promedio ponderación o nota por establecimiento de procedencia

Aun así, los alumnos procedentes de establecimientos educacionales particulares pagados lideraron la ponderación, destacando que fueron minoría en la muestra para el estudio.

Ahora bien, de los cinco ámbitos considerados para la obtención de datos, hay que mencionar que cuatro de ellos están compuestos por la resolución de 10 ítems que forman parte de un todo; sólo el ámbito de tecnología consideraba siete ítems.

Para seguir con la idea anterior, a partir del gráfico n. ${ }^{\circ} 5$ que se presenta a continuación, se ofrece información visual respecto de los cinco ámbitos declarados (Información, Comunicación y Colaboración, Convivencia Digital, Tecnología y Otras competencias digitales) y para cada uno de ellos, el mínimo y máximo de número de respuestas alcanzadas para cada una de ellas. Es así como para el ámbito de Información la puntuación máxima alcanzo a ocho y un mínimo de cinco respuestas resueltas; el ámbito presentaba un número de 10 ítems a resolver, donde el puntaje máximo estaba asociado a este valor. 


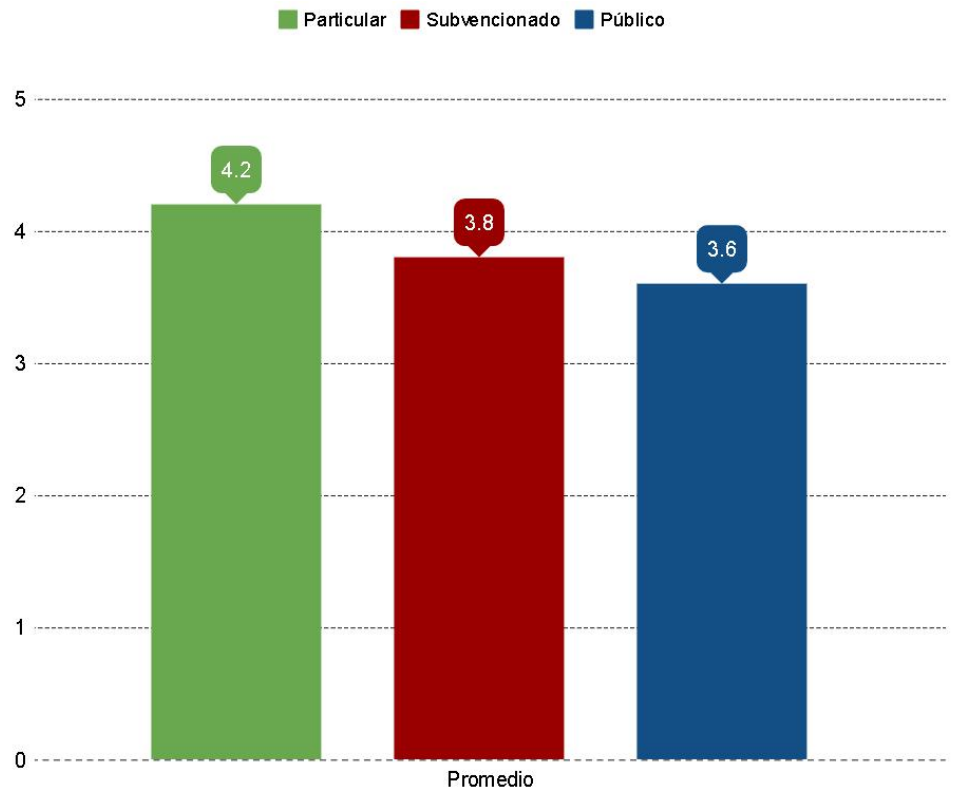

Gráfico. 5. Media, moda y máximo de acuerdo con las respuestas

Para el ámbito de "Comunicación y colaboración", es posible indicar tal como se muestra, que se logra el puntaje máximo de puntuación 10, y una mínima de cinco respuestas resueltas, donde se consideraban aquí 10 ítems a evaluar.

Precisamente el ámbito de "Tecnología" es el que alcanza la máxima de respuestas resueltas por un alumno, así también la mínima de la misma, siendo una contradicción muy interesante. Aun así, es necesario considerar que no se muestran tendencias, sino solamente respuestas alcanzadas; destacando que, de los 405 participantes evaluados, solo tres alcanzaron la puntuación máxima del ámbito. Los ámbitos con mayor número de ítems resueltos y, por tanto, deja entender que son en los que mejor se desarrollan los alumnos, tienen que ver con "Información". Aun así, considerando que la media alcanza el mismo valor que para la moda, parece encontrarse descendida en términos evaluativos. Si consideramos que al resolver los 10 ítems logramos la nota máxima (nota 7.0 en sistema chileno), entonces podemos inferir que con una media de cinco (traducido a nota chilena 3.5), no alcanzamos la nota mínima que debe ascender a 4.0. Por tanto, no estaría logrando la habilidad de acuerdo con el sistema evaluativo declarado para la prueba.

Es interesante notar que el ámbito de "Convivencia Digital" es el que se ha resuelto con mayor dificultad, o al menos, el que menos valoración alcanza en términos de respuestas logradas.

En general, el gráfico n. ${ }^{\circ} 5$ permite ver que las ponderaciones alcanzadas para la moda y la media en función del máximo que se indica para cada ámbito, hace notar la falta de anclaje de los dominios en general. Esto no se interpreta desde el punto del investigador como una tendencia negativa, pero sí permite ejercer ciertas reflexiones que dan luces a que es necesario instalar, reaprender, conceptos, habilidades y estrategias de resolución de problemas basados en TIC con el uso de herramientas y estrategias permitidas por ellas. 


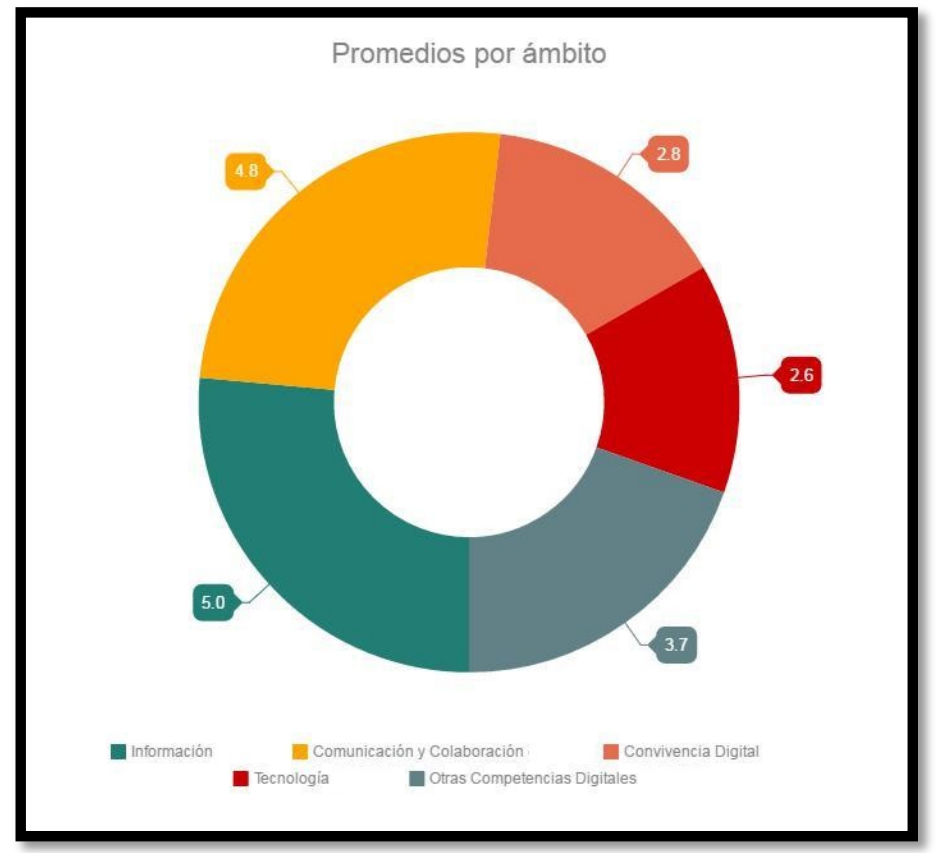

Gráfico. 6. Promedios por ámbitos

El gráfico n. ${ }^{\circ} 6$ muestra los promedios obtenidos para cada ámbito y a partir de las notas alcanzadas por los alumnos, que se desarrollan más ampliamente los conceptos de Información y Comunicación y colaboración.

Al repasar los dominios competenciales que suponen estos ámbitos, se deduce que trata de las actividades más usuales en la sociedad actual. Por diversos motivos u objetivos, como por ejemplo, buscar, evaluar y organizar información a partir de la necesidad de información, de manera de generar un nuevo producto de información, como así también comunicarse y saber compartir información en diversos formatos, entre otros. Por otra parte, los ámbitos más descendidos, según nos ofrecen los datos obtenidos a partir de la prueba, tienen que ver con "Tecnología" y "Convivencia digital", donde como nota promedio no superan 4.0. Esto es posible de traducir señalando que los estudiantes no alcanzan a manejar el $60 \%$ de las habilidades medidas para este ámbito.

Al observar la ponderación alcanzada para "Tecnología", es posible notar que esto tiene que ver más bien con un bajo nivel de manejo de conocimientos específicos más que con la habilidad en los usos, entendiendo que el mundo de las TIC es bastante dinámico. Por tanto, los dispositivos y nombres asociados a ellos varían constantemente, no manejar o mantenerse actualizado respecto de lo anterior repercute en la alta probabilidad de no tener la habilidad de resolver situaciones ante problemas técnicos. Así también, situándonos en el ámbito de "Convivencia digital" la baja ponderación se traduce a una falta de manejo por parte de los estudiantes de conocer y aplicar ciertos aspectos éticos, culturales y legales que rige y supone el uso de las TIC, además de los riesgos que ello implique, sobre todo al trabajar y participar activamente de procesos de colaboración en los diversos ambientes que se presentan. 


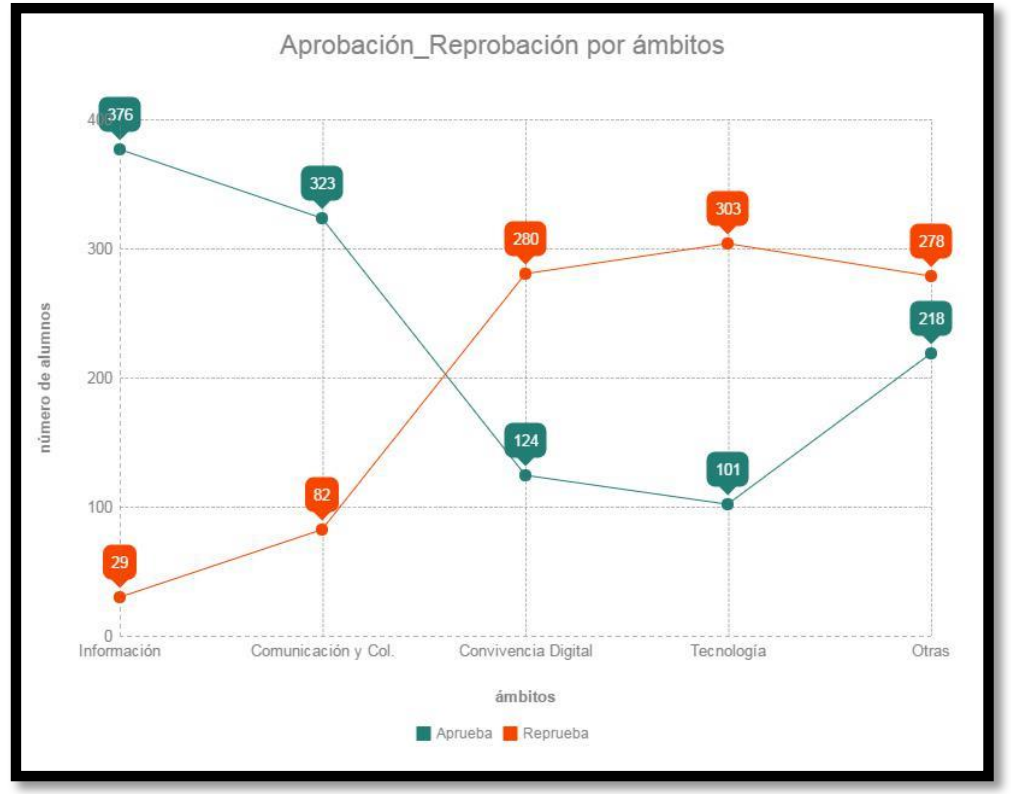

Gráfico. 7. Aprobación reprobación por ámbitos

Tal como reportan los resultados, y atendiendo a ellos, es que se establecerá foco y mayor atención a aquellos ámbitos que suponen un bajo rendimiento (Convivencia digital y Tecnología) a modo de sugerir potenciarlos durante el proceso.

Para finalizar y a modo de resumen que aporte a la construcción del estándar, se entiende que, instalados los cuatro ámbitos de la Matriz HTPA en los estudiantes, estos se encontrarían en posesión de los conocimientos y habilidades básicas y necesarias para participar, avanzar y tener la capacidad de adaptarse en el mundo actual que ofrece la sociedad del conocimiento.

Para los ámbitos de "Información" y "Comunicación y Colaboración", las actividades propuestas agrupaban necesariamente estar en posesión de habilidades que permitieran resolver actividades consistentes en el uso de TIC como medio para trabajar con la información como una fuente. Y por otro lado, usar las TIC para procesar la información y construir un producto que finalmente pueda ser compartido o transmitido, los cuáles evidenciamos fueron bien comprendidos y aceptados por los alumnos.

El ámbito "Información", precisamente en sus sub-ámbitos Fuente y Producto, requerían de actividades y procesos cognitivos propios de las Competencias Informacionales, de manera paralela y dinámica. Es claro que cuando se investiga en cualquier etapa del proceso formativo, aún por ocio, se deben generar búsquedas de información, donde procesualmente se seleccionen y se filtren datos según los intereses perseguidos.

Por su parte, las dimensiones de "Convivencia digital" y "Tecnología", en el desarrollo de la prueba se consideraron como aspectos transversales a los dos primeros ámbitos ya mencionados y así también un apartado conceptual básico. Por este motivo, las actividades relacionadas al desarrollo y trabajo con información, así como con la comunicación de la misma, implicaban relacionarse con herramientas que permitan dar solución a lo propuesto, comunicarse y vincularse con sus pares, navegar y consultar de ser necesario. También se presentan acciones que significaban al estudiante resolver dilemas éticos, entre otras posibilidades, todo esto como parte de las habilidades; todas las actividades a desarrollar 
requieren de un dominio conceptual y funcional de las TIC y la capacidad para resolver problemas ante situaciones emergentes; es aquí donde deberá de poner foco y preocupación, así potenciar las falencias y levantar en nuestros estudiantes las habilidades necesarias.

\section{Conclusiones}

Primeramente, comentar que la UMAG comprende los cambios y la dinámica por la cual transcurre un ciudadano en sociedad, en la cual nos corresponde navegar, y a veces naufragar, y que las necesidades y competencias claves que son de importancia desarrollar en un proceso formativo que considera las inclusiones y exclusiones de la vida misma para instalarse con comodidad y participar de ella. Es así como a partir de esta investigación, de los resultados arrojados y descritos a partir de la prueba de suficiencia aplicada a los alumnos/as de primer ingreso a la vida universitaria, es que se sugiere la necesidad de elaborar un concepto propio de competencia digital, que sea institucional, desde donde se pueda levantar y dada la necesidad urgente, de contar con un estándar de competencia digital para sus estudiantes en formación profesional, atendiendo a lo que conceptos como internet, globalización, colaboración, cultura digital entre otras, que se han generalizado.

Se considera de importancia anclar un estándar orientador que considere experiencias desde el mundo académico, sin olvidar lo que la Universidad de Magallanes (2010a) entiende por educación, y donde explicita y deja patente su compromiso de formar profesionales con las competencias requeridas para insertarse eficazmente en el mundo laboral, donde enfatiza su formación educativa basada en un enfoque por competencias, la cual traduce a la capacidad para actuar eficazmente en un conjunto de situaciones dadas. Por tanto, no se trata de aptitudes para demostrar conocimientos 0 talentos, sino más bien de entregar conocimientos, el desarrollo de habilidades y, lo más importante, saber qué hacer con ello en situaciones clave, una actitud frente a un problema y su resolución a partir de los conocimientos y herramientas que les han sido entregadas durante su formación, en este caso de las TIC y su inclusión en la vida diaria (ocio, profesionalización y formación).

La UMAG se encuentra preocupada de que el perfil de egreso de un profesional de esta institución se encuentre compuesto de competencias tanto genéricas (transversales a toda profesión) como específicas (definen acciones concretas de una determinada profesión). Intentando dar un sello formativo es que cuenta además con una serie de Competencias Sello genéricas para los estudiantes de la UMAG (Universidad de Magallanes, 2010a) donde es posible encontrar la competencia habilidades en el uso de las tecnologías de la información y comunicación, seleccionada entre otras ocho competencias que se entienden imprescindibles para la formación de todo egresado de la casa de estudios. Estas competencias han sido consideradas institucionalmente como un componente esencial y de valor de los egresados, por lo tanto, es vital actualizar esta competencia, entregarle una concepción, revalorar sus niveles de desempeño y a partir de ello levantar una propuesta de Estándar de Competencia Digital contextualizada y pertinente.

Finalmente, para dar respuesta al objetivo y pregunta de investigación ¿qué tan preparados se encuentran los estudiantes que ingresan a la vida universitaria en la Universidad de Magallanes en Chile respecto de las competencias digitales?, se encontró que en consideración a los ámbitos y habilidades de la Matriz HTPA, contemplados por el sistema educativo chileno, como parte del currículo nacional, estos se encuentran descendidos, y así lo revela el segmento evaluado, que no se encuentra preparado en éstas competencias. Es importante regular y velar por que en el proceso escolar anterior 
al universitario se trabajen las recomendaciones ministeriales, es urgente potenciar el sistema previo al universitario y nivelar durante la formación profesional en la universidad, en el contexto de armonización que pretende mantener esta casa de estudios y contextualizada en la región de Magallanes, en la Patagonia Chilena.

\section{Referencias}

Ala-Mutka, K., Punie, Y. y Redecker, C. (2008). Digital competence for lifelong learning (No. 48708). Sevilla: JRC. Recuperado de http://ftp.jrc.es/EURdoc/JRC48708.TN.pdf

Ala-Mutka, K. (2011). Mapping Digital Competence: Towards a Conceptual Understanding. Sevilla: European Commission, Joint Research Centre, Institute for Prospective Technological Studies.

Carretero, S., Vuorikari, R., y Punie, Y. (2017). DigComp 2.1: The Digital Competence Framework for Citizens with eight proficiency levels and examples of use (No. EUR 28558 EN). Luxemburgo: Unión Europea. Recuperado de https://op.europa.eu/en/publication-detail//publication/3c5e7879-308f-11e7-9412-01aa75ed71a1/language-en

ENLACES. (2015). SIMCE TIC. Recuperado de http://www.enlaces.cl/evaluacion-de-habilidadestic/simce-tic/presentacion/

Ferrari, A. (2013). DIGCOMP: A Framework for Developing and Understanding Digital Competence in Europe. European Commission. Recuperado de http://digcomp.org.pl/wpcontent/uploads/2016/07/DIGCOMP-1.0-2013.pdf

Guba, E. G. (1981). Criterios de credibilidad en la investigación naturalista. En J. Gimeno y A. I. Pérez (Comps.), La enseñanza: su teoría y su práctica (pp. 148-165). Madrid: Akal.

Hernández, R., Fernández, C. y Baptista, P. (2014). Metodología de la investigación (6.a ed.). México DF: Mc Graw Hill.

Ilomäki, L., Paavola, S., Lakkala, M. y Kantosalo, A. (2016). Digital competence-an emergent boundary concept for policy and educational research. Education and Information Technologies, 21(3), 655-679.

Ministerio de Educación. (2009). Estándares TIC para la Formación Inicial Docente en el contexto chileno: Estrategias para su difusión y adopción. Santiago, Chile: Ministerio de Educación, Centro de Educación y Tecnología del Ministerio de Educación de Chile (ENLACES) /UNESCO.

Ministerio de Educación. (2012). Actualización de Competencias y Estándares TIC para la Profesión Docente. Santiago, Chile: Ministerio de Educación, Centro de Educación y Tecnología del Ministerio de Educación de Chile (ENLACES).

Ministerio de Educación. (2013a). Matriz de Habilidades TIC para el Aprendizaje. Santiago, Chile: Ministerio de Educación, Centro de Educación y Tecnología del Ministerio de Educación de Chile (ENLACES). Recuperado de http://www.enlaces.cl/download/matriz-de-habilidades-tic-para-elaprendizaje/?wpdmdl=2134

Ministerio de Educación. (2013b). SIMCE TIC, Sistema Nacional de Medición de Competencias TIC en Estudiantes. Documentación Técnica 2013. Santiago, Chile: Ministerio de Educación, Centro de Educación y Tecnología - ENLACES. Recuperado de http://www.enlaces.cl/wpcontent/uploads/Documentacion_tecnica_SIMCE_TIC-2013_v10.pdf

OCDE. (2010). Habilidades y competencias del siglo XXI para los aprendices del nuevo milenio en los países de la OCDE. Madrid: Autor. 
Parlamento Europeo y Consejo de la Unión Europea. (2006). Recomendación del Parlamento Europeo y del Consejo de de 18 de diciembre de 2006 sobre las competencias clave para el aprendizaje permanente (2006/962/CE). Diario Oficial de la Unión Europea 30.12.200. Recuperado de https://eur-lex.europa.eu/legal-content/ES/TXT/PDF/?uri=CELEX:32006H0962\&from=ES

Sabariego, M. (2004). El proceso de investigación. En R. Bisquerra (Coord.), Metodología de la investigación educativa, (pp. 128-163). Madrid: La Muralla.

Sánchez-Antolín, P., Andrés-Vilora, C. y Paredes-Labra, J. (2018). El papel de la familia en el desarrollo de la competencia digital. Análisis de cuatro casos. Digital Education Review, (34). Doi: https://doi.org/10.1344/der.2018.34.44-58

UNESCO. (2017). La Educación para Todos (EPT). Recuperado de https://unesdoc.unesco.org/ark:/48223/pf0000127583_spa

Universidad de Magallanes. (2010a). Competencias Sello. Punta Arenas: Universidad de Magallanes.

Universidad de Magallanes. (2010b). Decreto n. ${ }^{\circ}$ 04/S/2010 Oficializa acuerdo de la honorable junta directiva que aprueba implementación asignatura "Computación Básica", a contar del año 2010. Punta Arenas: Universidad de Magallanes.

Universidad de Magallanes. (2010c). Proyecto Educativo Institucional de Universidad de Magallanes. Punta Arenas: Universidad de Magallanes. Recuperado de http://www.umag.cl/academicos/wpcontent/uploads/2015/09/Proyecto-Educativo-PEI-imprenta.pdf

Universidad de Magallanes. (2014). Decreto n.o 010SU2014 Oficializa nuevo Proyecto Educativo Institucional. Punta Arenas: UMAG.

Villa, A. y Poblete, M. (2011). Evaluación de competencias genéricas: principios, oportunidades y limitaciones. Bordón, 63(1), 147-170. 